\title{
ON SOME LINKS BETWEEN SYMBIOTIC STARS AND PLANETARY NFBULAE
}

\author{
LAURITS LEEDJÄRV \\ Tartu Astrophysical Observatory, EE2444 Tõravere, Estonia
}

\begin{abstract}
Some correlations found between different physical characteristics of symbiotic stars and planetary nebulae are discussed from the evolutionary point of view.

Symbiotic stars are considered as interacting binary stars, consisting of a red giant and a hot component which ionizes the stellar wind from the former, creating in this way physical conditions similar to those in planetary nebulae. Symbiotic stars are divided into types $\mathrm{S}$ and $\mathrm{D}$, containing, respectively, a normal red giant or a Mira as the hot component. In present paper some physical characteristics of the gaseous nebulae in symbiotic systems have been estimated by fitting the observed continuous spectra of symbiotic stars to the computed ones in a wide wavelengih range. Comparison of the obtained quantities to the compiled data for planetary nebulae demonstrates e.g. similar linear correlation between $\log M_{\text {gas }}$ and $\log n_{e}$ for both kind of objects (see Leedjärv, 1990 for more details). Also, it is found that the observed radio flux at $6 \mathrm{~cm}$ shows the tendency of decreasing when $n_{e}$ is increasing. When plotting these correlations, it turns out that D-type symbiotic stars occupy an intermediate position between S-type symbiotic stars and planetary nebulae. This can be explained by the different mass loss rates from Miras and from normal red giants. Miras in D-type symbiotic stars are quite near to the stage of planetary nebula, but probably it is not strictly correct to consider some symbiotic stars (V1016 Cyg, HM Sge) as protoplanetary nebulae. In planetary nebulae outer layers of the star are ionized by the core of the same star, while in symbiotic systems the source of ionizing radiation is another hot star. Further study of evolutionary links between symbiotic stars and planetary nebulae would yield a more detailed view in general evolutionary scheme of stars.
\end{abstract}

\section{References}

Leedjärv, L., 1990, Astrophysics , 32, 6. 